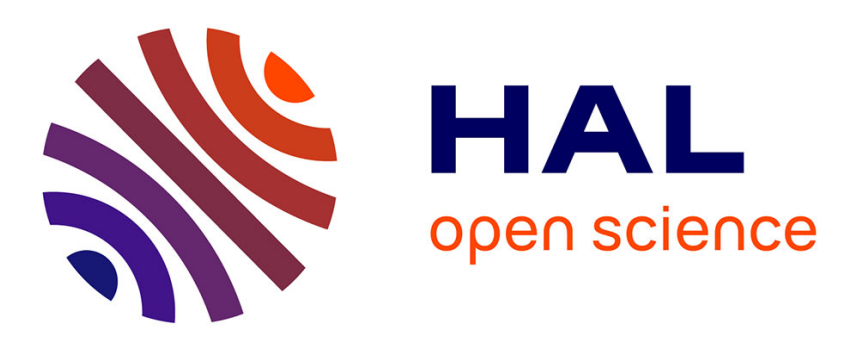

\title{
Large eddy simulation of turbulent heat transfer in pipe flows of temperature dependent power-law fluids
}

Paulin Sourou Ganmbode, Meryem Ould-Rouiss, Xavier Nicolas, Paolo

Orlandi

\section{- To cite this version:}

Paulin Sourou Ganmbode, Meryem Ould-Rouiss, Xavier Nicolas, Paolo Orlandi. Large eddy simulation of turbulent heat transfer in pipe flows of temperature dependent power-law fluids. M. Deville et al. (eds). Turbulence and Interactions. TI2018, 149, Springer, pp.123-129, 2021, Notes in Numerical Fluid Mechanics and Multidiscipliary Design. hal-02642264

\section{HAL Id: hal-02642264 \\ https://hal.science/hal-02642264}

Submitted on 28 May 2020

HAL is a multi-disciplinary open access archive for the deposit and dissemination of scientific research documents, whether they are published or not. The documents may come from teaching and research institutions in France or abroad, or from public or private research centers.
L'archive ouverte pluridisciplinaire HAL, est destinée au dépôt et à la diffusion de documents scientifiques de niveau recherche, publiés ou non, émanant des établissements d'enseignement et de recherche français ou étrangers, des laboratoires publics ou privés. 


\title{
Large eddy simulation of turbulent heat transfer in pipe flows of temperature dependent power-law fluids
}

\author{
Paulin Sourou Ganmbode ${ }^{1}$, Meryem Ould-Rouiss ${ }^{1}$, \\ Xavier Nicolas ${ }^{10000-0001-7161-6169]}$, and Paolo Orlandi ${ }^{2}$ \\ 1 Université Paris Est Marne-la-Vallée, MSME UMR 8208 CNRS, 5 Bd Descartes, \\ 77454 Marne-la-Vallée Cedex 2, France. \\ 2 Dipartimento di Ingegneria Meccanica e Aerospaziale, Università La Sapienza, Roma, Italy. \\ Meryem.ould-rouiss@u-pem.fr
}

\begin{abstract}
Heat transfer in turbulent forced convection of power-law fluids, in a heated horizontal pipe at isoflux conditions, is analyzed by large eddy simulations (LES), with an extended Smagorinsky model. A temperature dependent fluid is studied at various Pearson numbers $(0 \leq P n \leq 5)$, for two power law indices $(n=0.75$ and 1$)$, at Reynolds and Prandtl numbers $R e_{s}=4000$ and $P r_{s}=1$. The LES predictions are validated through comparisons with the literature at $P n=0$. They allow a better understanding of the physical mechanisms involved in the nonNewtonian temperature dependent fluid flows: with increasing $P n$, the relative viscosity is reduced close to the wall and enhanced towards the pipe center, reducing the turbulent fluctuations and heat transfer in the bulk and, as a consequence, the friction factor and Nusselt number.
\end{abstract}

Keywords: LES, non-Newtonian, temperature dependent power-law fluids, turbulence, pipe flow, heat transfer.

\section{Introduction}

Turbulent flows of non-Newtonian fluids are encountered in a wide range of engineering applications such as flows through ducts, pumps, turbines and heat exchangers, in the petroleum, chemical and food industries. When the Reynolds number is sufficiently large, large eddy simulation provides an effective tool to predict the effect of the flow parameters on the turbulent fields. Otha and Miyashita [1] developed a turbulence model that can reproduce DNS results in non-Newtonian fluid flows. They performed LES with a Smagorinsky model, extended according to their DNS results. They showed that this model can more accurately predict the velocity of turbulent flows, for fluids described by Casson's and power-law models, than the standard Smagorinsky model. Gnambode et al. [2] used this extended Smagorinsky model to predict the turbulent pipe flow of power law fluids, for various flow indices $(0.5 \leq n \leq 1.4)$, Reynolds numbers $\left(4000 \leq R e_{s} \leq 12000\right)$ and Prandtl numbers $\left(1 \leq P r_{s} \leq 100\right)$.

LES studies of heat transfer in a turbulent pipe flow of a non-Newtonian fluid are scarce. Some theoretical and experimental $[4,5]$ works focused on the 
turbulent heat transfer in pipe flows of non-Newtonian fluids for a constant viscosity case. Studies accounting for the temperature dependent viscosity observed in applications are even more scarce.

The objective of this work is to numerically investigate by LES, with an extended Smagorinsky model, turbulent heat transfer in the fully developed pipe flow of a temperature dependent power law fluid at $n=0.75$ and $1, R e_{s}=4000$, and $\operatorname{Pr}_{s}=1$, for $0 \leq P n \leq 5$, where the Pearson number, $P n$, is the dimensionless number measuring the temperature effect on the consistency of a non-Newtonian fluid. The aim is to gain more insights into such complex fluid flows whose viscosity is a function of both the temperature and the shear rate.

\section{Governing equations and numerical procedure}

The present study deals with the fully developed turbulent flow and heat transfer of power law fluids in pipes whose wall is heated at a constant heat flux $\phi_{w}$. The filtered non-Newtonian equations are made dimensionless using the centerline axial velocity of the analytical fully developed laminar profile, $U_{c l}=(3 n+$ 1) $U_{b} /(n+1)$, the pipe radius, $R$, and the reference temperature, $T_{r e f}=\phi_{w} R / \lambda$, for the velocity, length and temperature scales respectively, where $U_{b}$ is the bulk velocity and $\lambda$ the fluid thermal conductivity. The filtered equations (with the continuity eq. $\left.\partial \overline{u_{i}} / \partial x_{i}=0\right)$ read:

$$
\begin{gathered}
\frac{\partial \overline{u_{j}}}{\partial t}+\frac{\partial \overline{u_{i} u_{j}}}{\partial x_{i}}=\frac{\partial \bar{P}}{\partial x_{j}}+\frac{1}{R e_{s}} \frac{\partial}{\partial x_{i}}\left[\gamma^{-n-1} e^{P n \bar{\Theta}}\left(\frac{\partial \overline{u_{i}}}{\partial x_{j}}+\frac{\partial \overline{u_{j}}}{\partial x_{i}}\right)\right]+\frac{\partial \overline{\tau_{i j}}}{\partial x_{i}} \\
\frac{\partial \bar{\Theta}}{\partial t}+\frac{\partial}{\partial x_{j}}\left(\overline{u_{j}} \bar{\Theta}-\bar{\tau}_{\Theta j}\right)-\overline{u_{z}} \frac{d}{d z}<T_{w}>=\frac{1}{R e_{s} P r_{s}} \frac{\partial^{2} \bar{\Theta}}{\partial x_{k} \partial x_{k}}
\end{gathered}
$$

where $\Theta=\left(<T_{w}>(z)-T(r, \theta, z, t)\right) / T_{r e f}$ is the dimensionless temperature, $T_{w}$ is the wall temperature and $\langle>$ is an average in time and in the periodic directions. The subgrid heat flux tensor is defined by $\tau_{\theta j}=-\alpha_{t}\left(\partial \Theta / \partial x_{j}\right)$, with $\alpha_{t}=\nu_{t} / P r_{t}$ the turbulent thermal diffusivity linked to the turbulent viscosity, $\nu_{t}$, by the turbulent Prandtl number, $P r_{t}$, which is constant for a given flow index $n$. Preliminary LES carried out with the dynamical Smagorinsky model [2] resulted in the following estimates: for $n=1, P r_{t}=0.7$ and for $n=0.75, P r_{t}=1.5$. The Reynolds and Prandtl numbers of the simulations are defined as $R e_{s}=$ $\rho U_{c l}^{2-n} R^{n} / K_{0}$ and $P r_{s}=K_{0} / \rho \alpha R^{n-1} U_{c l}^{n-1}$. The apparent viscosity of the fluid is modeled by a power-law: $\eta=K \gamma^{n-1}$, where $K=K_{0} \exp \left[\operatorname{Pn}\left(\Theta-\Theta_{b}\right)\right]$, with $K_{0}$ the consistency at the bulk temperature $T_{b}, P n=b T_{\text {ref }}$ the Pearson number and $b$ the parameter of the thermo-dependence. $\gamma=\left(S_{i j} S_{i j}\right)^{1 / 2}$ is the shear rate, with the strain rate tensor $S_{i j}=\left(u_{i, j}+u_{j, i}\right) / 2$. The subgrid stress tensor is equal to $\overline{\tau_{i j}}=-2 \nu_{t} \overline{S_{i j}}$. In the non-Newtonian Smagorinsky model [1], $\nu_{t}=C_{s} f_{s}\left(f_{\eta} \Delta\right)^{2} \overline{S_{i j}}$, where $f_{s}$ is the van Driest wall damping function and $f_{\eta}=\eta / \eta_{w}$ the correction function for the change in viscosity.

An in-house finite difference code is used to solve the above LES model in a 3D cylindrical domain of axial length $L_{z}=20 R$ so that the largest thermal structures are well captured. Periodic boundary conditions (BC) and a uniform grid are used in $\theta$ and $z$ directions. No slip BC and a non-uniform grid refined close to 
the wall are used in the radial direction. The mesh size is $N_{\theta} \times N_{r} \times N_{z}=65^{3}$. More details on the non-Newtonian Smagorinsky model, numerical methods, simulation parameters and validations are given in [2].

\section{Results and discussion}

\subsection{Mean velocity and RMS profiles}

The mean streamwise velocity profiles, $U^{+}=U / U_{\tau}$, scaled by the friction velocity $U_{\tau}=\left(\overline{\tau_{w}} / \rho\right)^{1 / 2}$, are depicted in Fig. 1 left, for $R e_{s}=4000, \operatorname{Pn}=0$ and $\operatorname{Pr}_{s}=1$, as a function of the wall distance $y^{+}=\rho U_{\tau}(r-R) / \eta_{w}$ with $\eta_{w}=\left(K \gamma^{n-1}\right)_{w}$ the viscosity at the wall. These profiles are in satisfactory agreement with the DNS results by Rudman et al. [3] for $n=0.75$, and with the well-known universal law for $n=1$. The profiles of the root mean square (RMS) of the velocity fluctuations also agree well with the DNS data [3], Fig. 1 right. Since, for the shear thinning fluid $(n=0.75)$, the apparent viscosity is smaller close to the wall and larger in the bulk than with a Newtonian fluid $(n=1)$, the mean velocity is expected to be larger and the RMS to be smaller in the flow core (for $y^{+}>20$ ) for $n=0.75$.
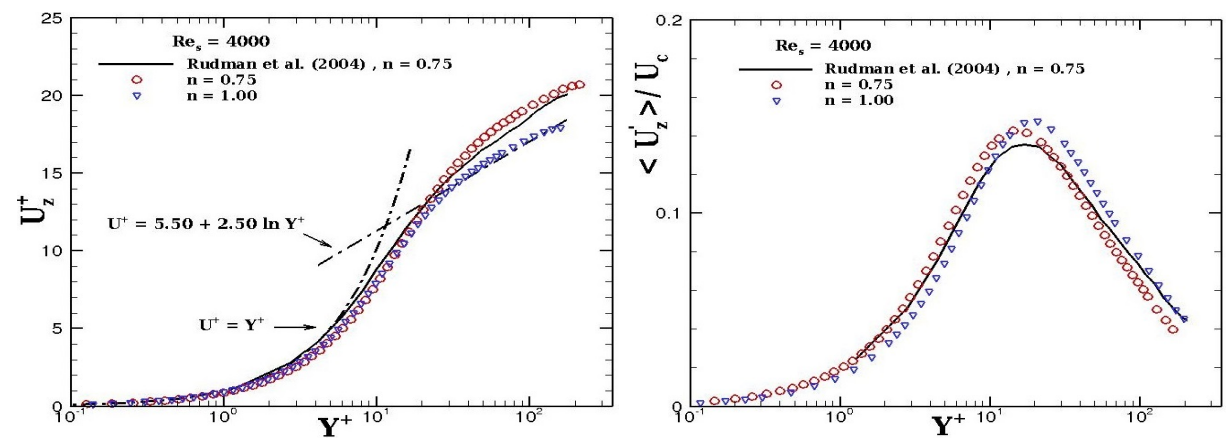

Fig. 1. Effect of $n$ on the axial velocity (left) and on the RMS of $U_{z}$ (right) at $P n=0$.

For the Newtonian fluid $(n=1)$, the viscosity is constant when $P n=0$ because $\eta=K_{0}$. In accordance with the experiments [4,5], for a $n<1$, the viscosity $\eta=K \gamma^{n-1}$ increases towards the duct center with increasing $P n$ (see Fig. 2 left, for $n=0.75)$. This is due to the decreasing shear rate $\gamma$ and the increasing consistency $K=K_{0} \exp \left[\operatorname{Pn}\left(\Theta-\Theta_{b}\right)\right]$ towards the center. Indeed, $P n>0$ and, when the duct wall is heated, $\Theta-\Theta_{b}=T_{b}-T$ is negative close to the wall but positive in the core region. The fluid becomes more rigid towards the pipe center and leads to a monotonous decrease of all the velocity fluctuations (normal and parallel to the wall) when $P n$ increases (see Fig. 2 right for $U_{z}^{\prime}$ ). Note that all the fluctuations are damped towards the pipe center for $P n \geq 3$.

On the other hand, a non-monotonous evolution of the mean axial velocity with increasing $P n$ is observed in the log-region at $n=0.75$ (Fig. 3 left) and $n=$ 1 (not shown). This behavior is correlated with the non-monotonous evolution of the mean wall shear stress $\overline{\tau_{w}}=K \overline{\gamma_{w}}$ or friction factor $f=2 \overline{\tau_{w}} / \rho \bar{U}^{2}$ (Fig. 3 
right): $f$ decreases and the flow accelerates when $P n$ increases from 0 to 2 and $f$ increases and the flow decelerates when $P n$ increases from 2 to 5 . This is due to the competition, in the wall boundary layer, between the decreasing consistency due to heating and the increasing shear rate when $P n$ increases.
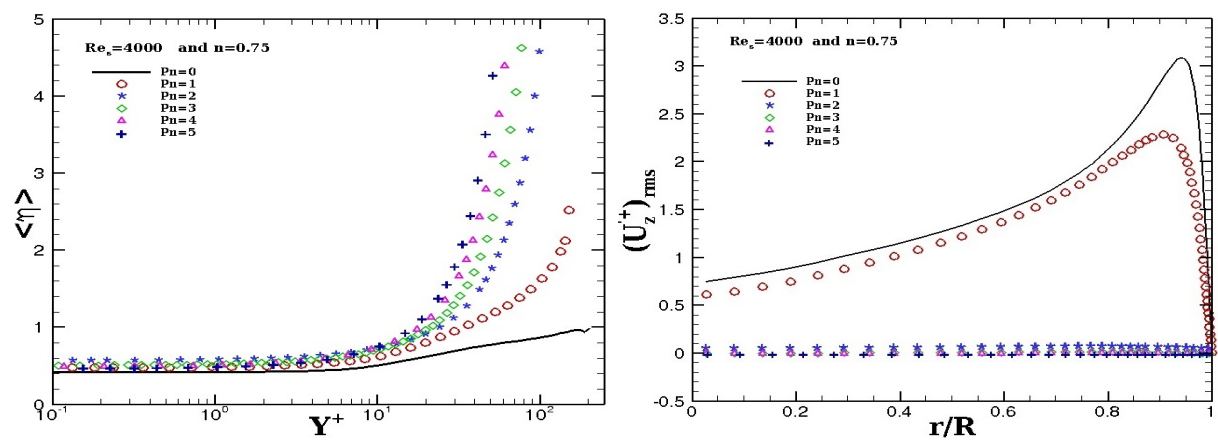

Fig. 2. Effect of $P n$ on the mean viscosity (left) and on the RMS of $U_{z}$ (right).
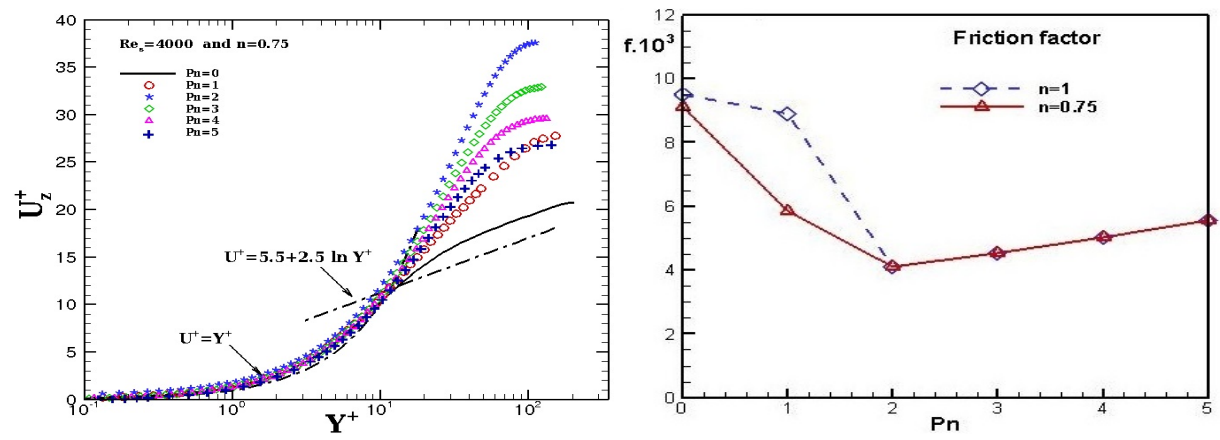

Fig. 3. Effect of $P n$ on the axial velocity (left) and on the friction factor (right).

\subsection{Temperature, RMS and turbulent heat fluxes}

As already seen above, due to the strong increase of the apparent viscosity in the bulk flow when $P n$ increases, the velocity and temperature fluctuations decrease from $P n=0$ to 2 and almost vanish beyond (Fig. 4 left). As a consequence, with increasing $P n$, the turbulent wall-normal heat flux undergoes a noticeable reduction (Fig. 4 right): it is strong at $P n=0$, twice smaller at $P n=1$ and nearly zero beyond due to the simultaneous decrease of the fluctuating radial velocity and temperature. The same behavior is observed for the axial turbulent heat flux $\left\langle U_{z} \Theta>\right.$ (not shown here).

To help interpreting Fig. 5 left, remind that $\Theta-\Theta_{b}=T_{b}-T$ is negative close to the wall and positive in the core region. Therefore the $\Theta$-increase corresponds to a reduction of $T$. Thus Fig. 5 left shows that the fluid is the hottest and the most $T$-homogenous at $P n=0$, thanks to the strong turbulent radial heat 
flux which controls heat transfer (Fig. 4 right). When $P n$ increases from 0 to 2, $T$ significantly decreases towards the pipe center due to the decrease of the turbulent radial heat flux and the smaller residence time of the fluid particles in the channel because of the fluid acceleration (Fig. 3 left). As a consequence the average Nusselt number, $N u=h D / k$, strongly decreases from $P n=0$ to 2 (Fig. 5 right). When $P n$ increases from 2 to 5 , the flow decelerates and heat transfer is controlled by the mean axial convection because the turbulent radial heat flux nearly vanishes: the residence time increases and the fluid is more heated by radial molecular diffusion (Fig. 5 left); thus, $N u$ is small and nearly constant (Fig. 5 right). The similar behavior of $f$ and $N u$ in Figs. 3 and 5 (right) also indicate a clear analogy between the momentum and heat transfer for each fluid.
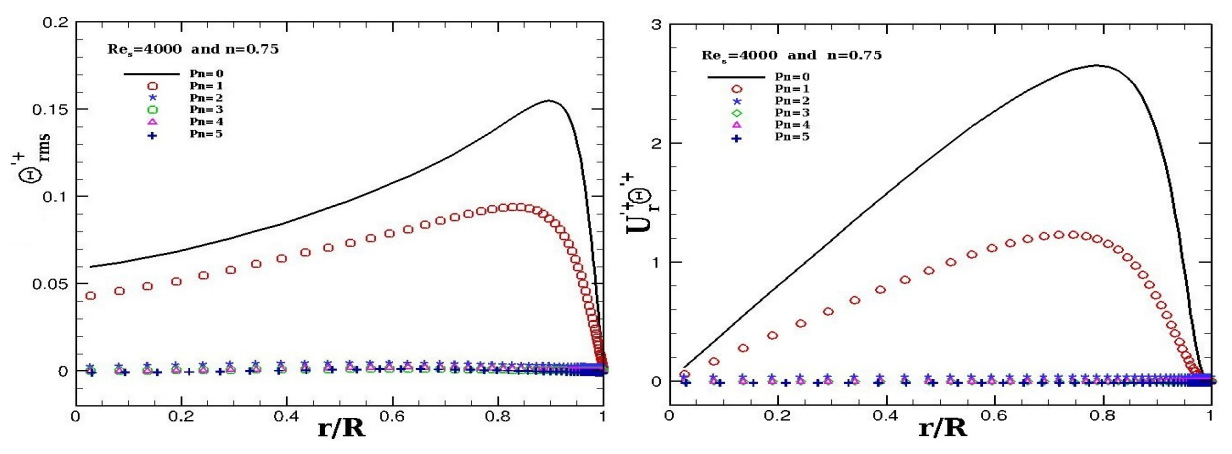

Fig. 4. Effect of $P n$ on the RMS of $\Theta$ (left) and on the radial heat flux (right).
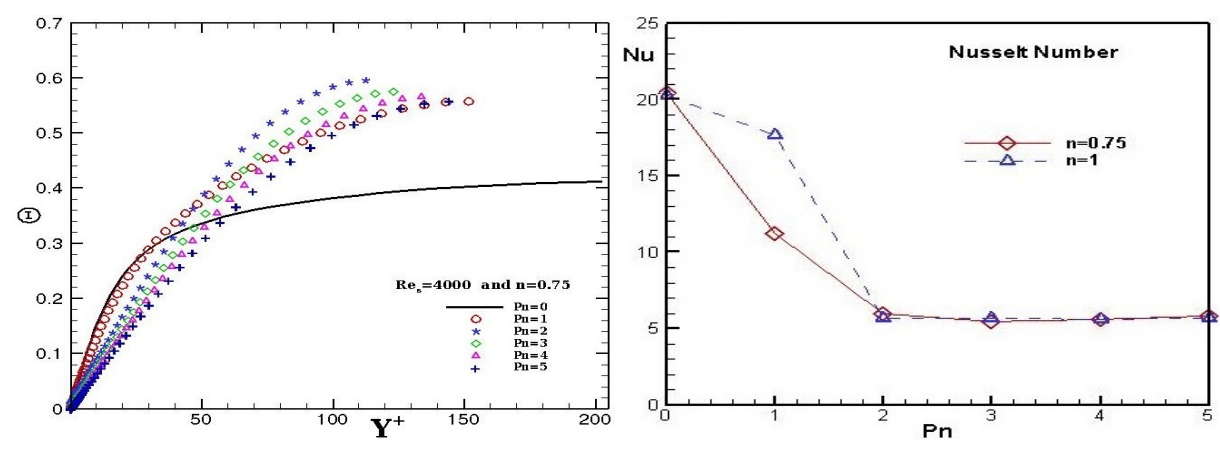

Fig. 5. Effect of $P n$ on the temperature (left) and on the Nusselt number (right).

\subsection{Visualizations}

To show the effects of $P n$ and $n$ on the flow, the resolved axial velocity fluctuations are presented in Fig. 6, in the full cylindrical plane $(\theta, z)$ at $y^{+} \simeq 15$. For the Newtonian fluid $(n=1)$, with increasing $P n$, the turbulent structures are less random and the streaks are larger indicating a less developed turbulence. For the shear-thinning fluid $(n=0.75)$, the number of streaks is reduced 
compared with $n=1$ and much longer streaks appear, particularly at $P n=1$. This suggests a reduction of the turbulence and, as a consequence, of the heat transfer, more pronounced at $n=0.75$ than at $n=1$ due the augmentation of the viscosity towards the pipe center. Clearly these visualizations and the $f$ and $N u$ behaviors in Figs. 3 and 5 indicate a structural change in the flow for $P n \geq 1$.
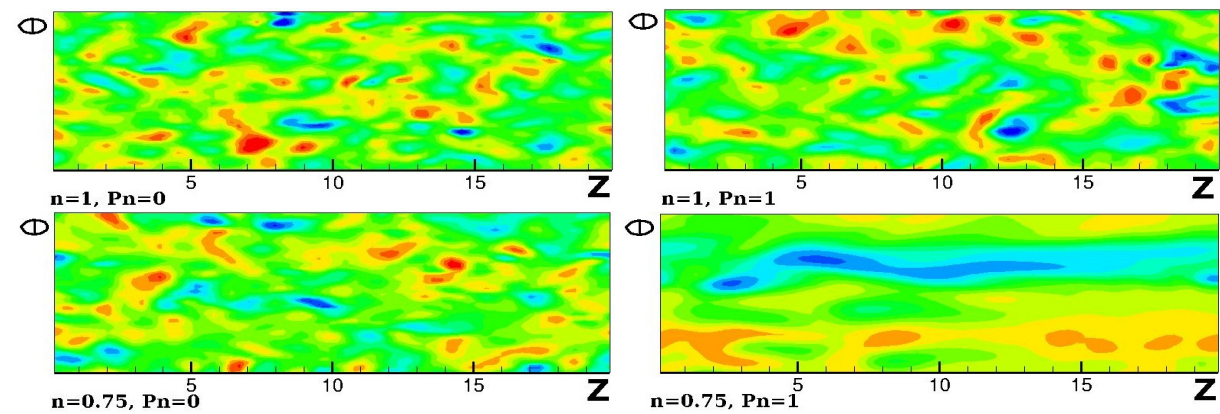

Fig. 6. $2 \mathrm{D}$ field of the resolved axial velocity fluctuations in the plane $(\theta, z)$ at $y^{+} \simeq 15$.

\section{Conclusions}

This study is the first contribution that uses LES, with an extended Smagorinsky model, to simulate turbulent flows and heat transfer of temperature dependent power-law fluids in pipes, under isoflux conditions. These LES enable to analyze these complex fluid flows with shear rate and temperature dependent viscosity. The Pearson number effect on the flow and thermal fields is in depth studied. For the non-temperature dependent viscosity case $(P n=0)$, the axial velocity profiles for the shear-thinning and Newtonian fluids, as well as the friction factors and Nusselt numbers, are in reasonably good agreement with the findings of the literature. With increasing $P n$ (temperature dependent viscosity case), the mean viscosity is enhanced towards the pipe center. As a consequence, the RMS of the temperature $\Theta^{+}$, similarly to the RMS of $U_{z}$, is reduced when approaching the pipe center, and the Nusselt number undergoes a significant reduction when $P n$ increases. From $P n=0$ to 2 , this leads to a decrease of the turbulent radial heat flux, acceleration of the flow and temperature reduction in the log-region.

\section{References}

1. Ohta, T., Miyashita, M.: DNS and LES with extended Smagorinsky model for wall turbulence in non-Newtonian fluids. J. Non-Newton. Fluid Mech. 206, 29-39 (2014).

2. Gnambode, P.S., Orlandi, P., Ould-Rouiss, M., Nicolas, X.: Large-eddy simulation of turbulent pipe flow of power-law fluids. Int. J. Heat Fluid Flow 54, 196-210 (2015).

3. Rudman, M., Blackburn, H.M., Graham, L.J.W., Pullum, L.: Turbulent pipe flow of shear-thinning fluids. J. Non-Newtonian Fluid Mech. 118, 3348 (2004).

4. Peixinho, J.: Contribution exp. à l'étude de la convection thermique laminaire, transitoire et turbulente pour un fluide à seuil ... PhD thesis, Univ. Nancy 1, France (2004).

5. Nguyen, V.T., Lebouché, M.: Etude num. de l'écoulement d'un fluide non-newtonien thermodépendant entre plaques parallèles. C.R.A.S. Mécanique 333, 365-369 (2005). 